\title{
Criterios y fundamentos para la implementación de Centros de Recursos para el Aprendizaje y la Investigación
}

\author{
Raquel Zamora Fonseca \\ Universidad de Cienfuegos - UCF, Cuba
}

\section{ANÁLISIS}

\begin{abstract}
Resumen
Revisa los criterios y fundamentos base para la implementación de Centros de Recursos para el Aprendizaje y la Investigación CRAI. El análisis esta enfocado a la implementación de CRAls en bibliotecas universitarias y a los modelos organizativos que se pueden adoptar.
\end{abstract}

Palabras clave

Centros de Recursos para el Aprendizaje y la Investigación ; CRAI ; Bibliotecas universitarias ; Aprendizaje ; Investigación

\section{Criteria and foundations for the implementation of the Learning Resource Centers}

\author{
Abstract \\ Review the criteria and rationale basis for the implementation of research - library and learning resource centers. The analysis \\ focused on the implementation of CRAls in university libraries and organizational models that can take.
}

Keywords

Learning Resources Centre ; LRC; University libraries ; Learning ; Research

\section{Introducción}

La American Library Association define la biblioteca universitaria como una biblioteca (o sistema de éstas) establecida, mantenida y administrada por una universidad, para cubrir las necesidades de información de sus estudiantes y apoyar sus programas educativos, de investigación y demás servicios (ALA glossary of Library and information Science, (1993, Citado en Martín, 2008).

La biblioteca, concebida hasta el momento como un servicio de apoyo a la universidad, ha de transformarse en un servicio indispensable clave que ayude y facilite a los estudiantes y profesores a acceder, gestionar y manejar la información en una nueva época llamada "Sociedad del conocimiento".

Uno de los retos actuales de la universidad es la planificación y gestión de los servicios universitarios que ofrecen a la comunidad, principalmente aquellos que se relacionan con la docencia, la investigación y la formación continuada a lo largo de toda la vida. Para conseguir este objetivo, las universidades buscan estrategias de mejora, idean propuestas organizativas y ponen en marcha programas y proyectos para conseguir una mayor eficacia de los recursos. 
La biblioteca universitaria hasta ahora considerada como un servicio clásico de la universidad, como tesorera de la producción científica y técnica de todos los conocimientos es quien en este nuevo entorno, puede aglutinar toda una serie de servicios de la comunidad universitaria y presentar soluciones favorables a la consecución de los cambios. La biblioteca con una imagen conservadora y poco amante de los cambios, se convierte en un espacio dinámico motivador, acogedor, placentero, abierto al aprendizaje con nuevos recursos tecnológicos.

Para el australiano Leonard Jolley, la biblioteca universitaria juega un papel diferente e indispensable en sintonía con el objetivo básico de la universidad, animando al estudiante a la búsqueda personal e individual del conocimiento y del saber. En cuanto al personal de la biblioteca, éste no deberá de interesarse tanto por las tareas rutinarias y administrativas sino que se deberá inclinar por la promoción del estudio y del conocimiento. La verdadera tarea del bibliotecario universitario es, por lo tanto, formar al estudiante para que se forme a sí mismo. (Martín, 2008).

Las Tecnologías de la Información y las Comunicaciones. (TIC), han permitido a las bibliotecas mejorar la gestión de la organización, almacenaje y acceso de la documentación tanto en soporte papel como electrónico. Las TIC permiten que se realice todo de una forma radicalmente distinta y a su vez han provocado nuevas políticas bibliotecarias que dan como resultado nuevos servicios bibliotecarios directos a los usuarios. La biblioteca universitaria se convierte, cada vez más, en un centro que está en un constante movimiento con el fin de satisfacer las necesidades de sus usuarios, los cuales cada día son más exigentes. Las nuevas formas de estudio, de lectura y de aprendizaje de los usuarios dan como resultado nuevos modelos de biblioteca universitaria.

Según Martínez (2005) el mundo universitario de EEUU, Reino Unido y Holanda países avanzados en la innovación educativa se han implementando nuevos modelos de biblioteca universitaria basados, sobre todo, en dos actuaciones:

- La transformación de la biblioteca presencial en un centro abierto durante amplios horarios con recursos disponibles para el aprendizaje de todo tipo. También se refuerza la idea social de encuentro y comunicación de la comunidad universitaria.

- La configuración de un nuevo equipamiento a partir de una fuerte apuesta tecnológica en sus servicios, con una biblioteca digital que aglutina los sistemas de información.

En torno a la nueva biblioteca se integran aquellos servicios clave para los profesores y los estudiantes que están ligados al desarrollo de sus proyectos educativos y relacionados con la información y las tecnologías. La biblioteca, en este nuevo contexto se llama "Learning Resources Centre" (Centro de Recursos para el Aprendizaje y la Investigación). La Biblioteca se convierte en un nuevo equipamiento donde el usuario puede encontrar otros servicios universitarios de manera integrada y sin tener que hacer largos desplazamientos. De esta manera, la biblioteca se convierte en el verdadero centro de los recursos educativos básicos para la comunidad.

La nueva biblioteca universitaria deja de llamarse biblioteca y pasa a llamarse Centro de Recursos para el Aprendizaje e Investigación CRAI. La Biblioteca es un centro de recursos para el aprendizaje, la docencia y la investigación y las actividades relacionadas con el funcionamiento y la gestión de la Universidad /Institución en su conjunto. La Biblioteca tiene como misión facilitar el acceso y la difusión de los recursos de información y colaborar en los procesos de creación del conocimiento, a fin de contribuir a la consecución de los objetivos de la Universidad /institucionales. Es competencia de la biblioteca seleccionar y gestionar los diferentes recursos de información con independencia del concepto presupuestario, del procedimiento con que hayan sido adquiridos o de su soporte material. (REBIUN, 2002, Citado en Balagué, 2003).

De acuerdo con Martínez (2005), la biblioteca se convertirá en un centro dinamizador del nuevo aprendizaje, en un centro social de la comunidad universitaria. La universidad que se arriesgue a optar por realizar estos cambios deberá de transformar la biblioteca a partir de un nuevo planteamiento. Un nuevo diseño de espacios y servicios para reunir otros elementos que antes se ubicaban fuera de la biblioteca. Equipar y definir un mobiliario adaptado no sólo para libros sino para estaciones de trabajo y así asegurar el acceso a la información digital, facilitar todo tipo de hardware y software, implementar horarios de abertura amplios, facilitar otros materiales y recursos diversificados, definir una nueva organización y unos nuevos procedimientos. 


\section{Los fundamentos de cambio}

Para Cabo ( 2007) estos cambios en la biblioteca unversitaria se fundamentan en dos aspectos base:

\section{a) La necesidad de eficiencia en la gestión universitaria:}

- Creciente demanda de nuevos servicios en un contexto económico

- Necesidad de crea estructuras más eficientes y más económicas.

- Racionalización de los presupuestos.

- Compartir espacios, ampliar horarios

- Aumentar y mejorar la prestación de servicios sin aumentar la plantilla.

\section{b) Las TIC y la innovación docente:}

- Papel esencial de las TICs en la innovación y renovación docente

- La tecnología es utilizada como renovadora de la actividad.

- Combinación de la enseñanza presencial con el soporte de plataformas tecnológicas.

- Aprendizaje mixto: combinación de sesiones presenciales con trabajos realizados con las tecnologías. (Web, correo electrónico, foros, videoconferencias)

- El estudiante ha de apoyarse en diferentes recursos, sobre todo digitales.

- Esta metodología facilita que el estudiante desarrolle un proceso de aprendizaje: más flexible, variado y adaptado a su propio ritmo.

Área (2005) afirma que "un CRAI es un servicio universitario que tiene como objetivo ayudar a profesores y estudiantes a facilitar las actividades de aprendizaje, de formación, de gestión y de resolución de problemas, ya sean técnicos, metodológicos o de conocimiento, en el acceso y uso de la información. Una diferencia importante entre un CRAI y una biblioteca universitaria es que, mientras que ésta se concibe como un depósito o almacén de documentos a la espera de ser consultados, aquel se adelanta y genera las necesidades potenciales de los usuarios en múltiples planos y aspectos. Como afirma Martínez (2004) "el nuevo modelo de biblioteca no tiene como centro el libro, sino el sujeto". Esta es una idea potente y nuclear del nuevo papel de las bibliotecas universitarias concebidas como centros de recursos: ser un servicio centrado sobre las necesidades de los alumnos, profesores e investigadores de la comunidad universitaria.

Martínez (2005) refiere que los CRAI han de posibilitar el acceso a toda la información y documentación que el usuario necesite de la universidad y debe hacerlo de forma fácil, rápida y organizada. Que ha de programar el crecimiento de las distintas colecciones bibliográficas, así como la integración de otros materiales y colecciones tanto en soporte papel como electrónico.

El CRAI tiene que disponer de un amplio abanico de servicios generales, pero también servicios adaptados, personalizados según las necesidades de los usuarios, tanto a tiempo completo como a tiempo parcial. El profesor tiene que encontrar en el CRAl el soporte tecnológico, los equipamientos adecuados, el personal pedagógico creativo y necesario y la información que necesita para formarse en las nuevas técnicas pedagógicas, el nuevo 
hardware y software docente, pero también el acceso, gestión y manipulación de la información. El profesor tiene que ver el CRAI como la nueva aula con multitud de servicios y recursos a su alcance.

EI CRAI debe facilitar a los estudiantes una experiencia de aprendizaje total mediante la interacción con libros, personas y tecnología. El CRAI ha de posibilitar que profesores y estudiantes puedan continuar colaborando en proyectos conjuntos. Por tanto debe disponer de espacios diferenciados para el estudio individual y en grupo, para realizar sesiones de trabajo, preparación de seminarios y presentación de proyectos. Todos los puntos de lectura deben tener acceso a la red, para que los usuarios puedan acceder a ella con su PC portátil o estar preparado con la WIFI (internet inalámbrico).

Área, (2005) plantea que desde su punto de vista, un CRAI en una institución de educación superior estaría definido por cuatro conceptos clave tal como representa el gráfico adjunto.

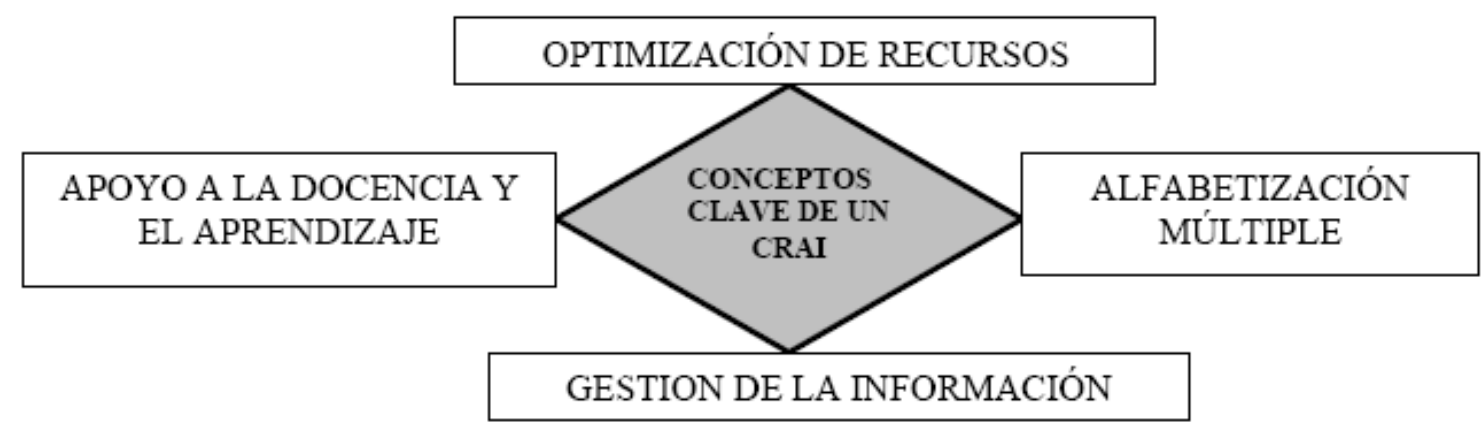

Figura 1. Cuatro conceptos claves de los CRAI en la Educación Superior. Tomado de: Área, 2005

Uno de los problemas más relevantes que padecen actualmente las universidades es la falta de coordinación y, en ocasiones, el solapamiento de los distintos servicios que presta. Esto provoca un gasto económico a la universidad y una sensación de falta de transparencia a los usuarios de dichos servicios. Si no están adecuadamente coordinados y en muchas ocasiones, centralizados en determinados espacios físicos, muchos servicios son desaprovechados en gran medida debido a la dificultad de acceder a ellos. Un espacio unificado como es el CRAI, permitirá optimizar tanto los recursos disponibles como la mejora de los recursos que se presta.

\section{Los factores necesarios}

Los factores que deben ser tenidos en cuenta a la hora de promover la convergencia de los servicios y crear un CRAI son diferentes para cada universidad, y dependen de su estructura, su historia, su cultura y sus metas organizativas. Sin embargo, para Nuria Balagué (2003) hay algunos aspectos comunes:

- Los servicios de la universidad tienen todas unas mismas misiones: dar soporte a la docencia, el aprendizaje y la investigación. Los CRAI realizan una excelente contribución a dicha misión.

- El nuevo paradigma educativo hace que las universidades se replanteen, no únicamente la docencia, sino todos aquellos elementos que le dan soporte. Los CRAI ayudan a la innovación docente.

- Las posibilidades que, para la gestión de la información, brindan las tecnologías de la información, son amplias y en constante cambio. La convergencia de servicios en el seno de los CRAI permite desarrollar una estrategia al respecto. 
- Un modo de mejorar la asistencia a estudiantes, profesores e investigadores es ofrecerles un único servicio capaz de ayudarles a resolver problemas diversos relativos a su actividad. Los CRAI permiten ofrecer servicios de mayor calidad, concentrados y adecuados a las necesidades de cada usuario.

Las Bibliotecas convertidas en CRAI deben ser el componente esencial del nuevo modelo en el proceso educativo que requiere la sociedad del conocimiento. Las bibliotecas en un nuevo modelo de aprendizaje organizan y representan la información y el conocimiento en diferentes formatos (DVD, CD, Web), lo que apoya a los estudiantes en sus estudios e investigaciones. Los CRAI, propician el trabajo en equipo, la creatividad y desarrolla su autonomía en la búsqueda de información; así como en la elaboración de trabajos prácticos. Les permite a los profesores elaborar sus propios materiales didácticos para el apoyo a la docencia, ofrece la posibilidad de que el profesor disponga de un aula donde puede montar su asignatura en plataformas de aprendizajes. Los profesores tendrán la posibilidad de que sus alumnos intercambien directamente con diferentes materiales que serán de utilidad indispensables en sus clases, como es el caso de la asignatura Metodología de la Investigación o la Introducción a la Información. Indudablemente este nuevo modelo de Bibliotecas del siglo XXI, se hace necesario e indispensable para enfrentar este nuevo proceso de enseñanza y aprendizaje, donde los estudiantes juegan un rol protagónico, donde participan estudiantes que son nativos de la era digital y se les hace ineludible ya la convergencia de los servicios de biblioteca tradicional con los servicios informáticos, es decir los servicios y posibilidades que ofrece la TICs.

Por ello se hace necesario el estudio de los diferentes modelos organizativos de convergencia del CRAI, para su implementación en la Educación Superior. Andrew McDonald, profesor y Director de Servicios bibliotecarios y para el aprendizaje de la University of East London, ha destacado recientemente las cualidades que debe tener un buen espacio para el aprendizaje, ya sea en edificios nuevos o reformando el espacio existente. Estas cualidades son lo que ha hecho suyas la Sección de Edificios y Equipamientos de IFLA publicada "IFLA Library Building Guidelines, obra que aporta información, puntos de vista y re-flexiones actualizadas sobre la planificación de bibliotecas. (Martín, 2009).

Un buen espacio para el aprendizaje debería ser:

- $\quad$ Funcional espacio que funcione bien, sea atractivo y dure mucho.

- Adaptable espacio flexible cuyo uso se pueda cambiar con facilidad.

- Accesible espacio social que sea acogedor, fácil de usar y estimule la inteligencia.

- Variado con diferentes entornos y soportes para el aprendizaje.

- Interactivo espacio bien organizado que fomente el contacto entre los usuarios y los servicios.

- Favorable espacio humano de gran calidad que inspire a la gente.

- Adecuado al medio ambiente con las condiciones apropiadas para los lectores, los libros y los ordenadores.

- Adecuado para la tecnología de la Información con unas prestacionesflexibles para los usuarios y el personal.

\section{La transformación de la biblioteca universitaria}

El nuevo modelo en que se inspira la transformación de la biblioteca universitaria es el de CRAI, surgido en el Reino Unido en los años ochenta. 
Según Nauto (2006), las universidades británicas de Bath, Braford, East London, Glamorgan, Hertfordshire, Leeds Metropolitan, London Guidhall, Luton y Sheffield Hallam son las primeras en crear Centros de Recursos para el Aprendizaje, estos centros responden a una estructura que integra:

- Recursos bibliotecarios

- Recursos tecnológicos

- Recursos audiovisuales

- Sistemas de información

- Instalaciones y medios para la edición electrónica

- Creación de materiales interactivos

Este nuevo modelo de organización y funcionamiento subyace en los centros de Recursos Para el Aprendizaje y la Investigación (CRAI) españoles, basados en los Integrated Learning Centers anglosajones.

Desde hace casi una década, los servicios bibliotecarios han experimentado procesos de transformación derivados de la irrupción de las nuevas TIC y especialmente Internet en el entorno de la biblioteca. Esta ya no conoce fronteras, extendiéndose más allá de los límites del propio espacio físico y evolucionando hacía nuevas formas abiertas, flexibles y marcadas por estos nuevos escenarios:

- Nuevos modelos de enseñanza y aprendizaje.

- Integración de las TICs en los sistemas educativos.

Ante estos cambios, la biblioteca ha de innovar y adaptar sus servicios a los nuevos contextos, necesidades y retos del aprendizaje. Se apuesta por una convergencia de servicios, basada en la idea de futuro de los sistemas digitales integrados de información orientados al usuario, capaces de suministrar informaciones analógicas y numéricas, fuentes documentales tradicionales y electrónicas, así como servicios de asesoramiento indispensables para explotar esas fuentes.

En el ámbito organizativo de las bibliotecas ya se está trabajando en proyectos transversales con los servicios informáticos, con los servicios audiovisuales, con los centros de idiomas, con los servicios de atención al estudiante.

Los CRAI tienen como Misión facilitar el acceso y la difusión de los recursos de información y colaborar en los procesos de creación del conocimiento, a fin de contribuir a la consecución de los objetivos institucionales de la universidad.

El CRAI supone la evolución desde el clásico modelo de biblioteca como depósito y gestión de libros a un modelo integrado de información y servicios, dinámicos y flexible, que proporcione soporte al aprendizaje, poniendo a disposición de los estudiantes documentos pertinentes para su desarrollo educativo, ofreciéndoles asesoramiento y formación. También ha de ser un laboratorio de producción de contenidos en el que los profesores puedan crear y experimentar sus materiales docentes y los estudiantes puedan elaborar presentaciones y trabajos. En este punto de encuentro el CRAI dejaría de ser un futurible, transformándose en un referente importante para la educación informacional de profesores y estudiantes, convirtiéndose en facilitador para el desarrollo de habilidades y competencias transversales relacionadas con aprender a aprender, saber localizar, evaluar y sintetizar información para la resolución de problemas, aprender a trabajar de manera autónoma. (Balagué, 2003).

El camino hacia el CRAl es lento y laborioso, pues los enfoques, la cultura y las razones para la convergencia difieren entre las distintas universidades, debido a la propia dinámica de los servicios a la hora de delimitar sus 
fronteras, así como a las conservadoras estructuras organizativas subyacentes, que a veces son poco eficaces o muestran reticencias en momentos de cambios. La noción de convergencia, que es esencialmente anglosajona ha calado hondo en las universidades del Reino Unido. (Follet, 1993, Citado en Pinto, 2008a, p.57).

Pugh (1997, Citado en Pinto, 2008a:57) plantea que existe la creencia errónea de que la convergencia podría realizarse mediante la colaboración al margen de todo cambio de tipo administrativo o estructural. No obstante sabemos que no hay un procedimiento ni un modelo único para conseguirlo, sino más bien un gradiente de opciones. (Sidgreaves, 1995, Citado en Pinto, 2008a, p.57) que van desde la simbiosis entre servicios informáticos y biblioteca a un único macroservicio con un solo responsable, pasando por la creación de una estructura integrada de todos los servicios para los usuarios, pero con el mantenimiento de estructuras independientes para el resto de las actividades. El factor que justifica la convergencia es el deseo de la institución de concentrar los servicios de información y de apoyo a la comunidad universitaria para mejorar la eficacia y eficiencia del aprendizaje y de la gestión del conocimiento de la institución, definiendo una estrategia documental integrada que haga converger todas las fuentes de información presentes en el seno de la universidad. Esta nueva realidad pondrá una reorganización estructural de los trabajadores del conocimiento, que serán gestores y expertos en información especializada, conocedores de los modelos de gestión participativa y de las ventajas de la innovación del trabajo en equipo en un sector dinámico y creativo.

De acuerdo con Kazmer (2002, Citado en Pinto, 2008a, p.59) se puede decir que la convergencia de los servicios de apoyo a la docencia e investigación en un CRAI es una apuesta emergente y estratégica de las universidades para concentrar y rentabilizar sus servicios de apoyo a la comunidad universitaria, para potenciar el trabajo en equipos polivalentes que gestionen mejor la información y el conocimiento, para minimizar costes y para ser más competitivas y eficientes en la gestión, así como para contribuir en la educación informacional de la comunidad universitaria, especialmente de los estudiantes.

El proceso de convergencia y transformación en CRAI requiere tanto de un sólido compromiso de los responsables políticos y académicos que respalden la iniciativa de un proyecto emergente de calado en la organización como de una fuerte implicación y motivación de una gran diversidad de profesionales que habrán de trabajar en equipo (bibliotecarios, especialistas en información, informáticos. Técnicos multimedia, pedagogos, orientadores psicólogos sociales, editores y correctores.

Cada universidad ha de buscar la mejor fórmula para dotarse de un CRAI a su medida, que responda a su misión, metas y objetivos y que al tiempo sea un reflejo de la cultura institucional.

\section{Modelos de CRAI}

Pinto (2008a, p.61) plantea los siguientes modelos organizativos de convergencia del CRAI:

\subsection{Modelo Burocrático:}

Este modelo tiene una estructura jerárquica. Los servicios actúan de forma independiente, enlazados sólo por un mecanismo de control que hace llegar la información hasta la persona encargada de tomar decisiones. El trabajo es supervisado por un responsable que rinde cuentas a la dirección de la institución.

\subsection{Modelo Funcional:}

Es el más adecuado al pleno despliegue de los beneficios de la convergencia, tanto para los usuarios y el personal como para el desarrollo de la institución. Está basado en el funcionamiento de equipos organizados en torno a un dominio o grupos de dominios y reagrupados en una estructura sectorial integrada de servicios. Se emplean mecanismos integradores en el funcionamiento de los equipos, y los proyectos. El equipo de dirección puede verse reducido de forma importante. Dentro de los activos de este modelo de convergencia destacamos que:

a) Se atenúa la verticalidad de la estructura, pues a medida que los equipos ganan en garantía y se acrecienta el cambio cultural a favor de la integración, se van suprimiendo 
progresivamente los puestos relativos a la administración específica de los servicios (servicios de biblioteca o servicio informático, por ejemplo) y se nombran responsables encargados de una misión más global.

b) Los sistemas de comunicación son eficaces y engloban a los individuos, a los equipos, a las diferentes secciones y a la dirección. La información circula en los dos sentidos.

c) En el interior de los equipos, los responsables de los diferentes grupos forman una unidad de dirigentes intermediarios que aseguran la conexión con los distintos servicios de la organización. Las decisiones son tomadas por el conjunto del equipo y están centradas en el usuario y sobre todo en la calidad de los servicios suministrados.

d) Se concede una gran importancia a la difusión y al desarrollo del conocimiento y especialmente a la formación por competencias, sobre todo aquellas relacionadas con el saber hacer.

\subsection{Modelo de participación voluntaria:}

Es uno de los modelos de convergencia más interesantes, pues favorece la cooperación entre los distintos servicios de apoyo a la docencia, al aprendizaje y a la investigación.

Algunas universidades han apostado para que estos servicios sean gestionados en coordinación por un miembro del comité director de la institución, introduciendo relaciones más dinámicas que los clásicos mecanismos de responsabilidad unilateral. Como por ejemplo de este modelo se destaca la Universidad de Dervy (http://www.dervy.ac.uk) donde los servicios informáticos y de biblioteca han trabajado al unísono para la creación de un nuevo centro de recursos del conocimiento, basando la cooperación en el trabajo en equipo. También la Universidad de Tilburg (http://www.tilburguniversity.nl )que apuesta desde el año 2004 por una política de integración de los servicios de biblioteca, de informática y del centro multimedia (desde enero 2007) bajo la dirección de un único responsable.

Se puede hablar de tres modelos de CRAI, según los tipos de convergencia mencionados anteriormente:

a) Centralizado, con una concentración total de los servicios en un solo centro.

b) Descentralizado, con un despliegue de opciones: Unificación institucional acompañada de una diversificación de servicios en distintos centros; varios CRAI ubicados en cada uno de los campus de la institución.

c) Híbrido: basado en la propuesta de un solo CRAI, pero con servicios descentralizados.

La implementación de estos modelos de convergencia depende de numerosos factores: entre otros, el contexto y las características de la organización, las razones estratégicas y organizativas, la disponibilidad de recursos, la actitud hacia la gestión del cambio teniendo en cuenta su idiosincrasia, la universidad deberá elegir el modelo que mejor se adapte a sus circunstancias, misión y objetivos, pues la implantación del modelo deberá ir acompañada de una transformación de las estructuras administrativas y funcionales de los servicios convergidos que favorezca el camino hacia el CRAI, con un cambio sustancial en la descripción de los puestos de trabajo, en las funciones asignadas, en la definición de objetivos y en la evaluación de rendimientos.

McDonald (1995, Citado en Pinto 2008a, p.64) considera que la convergencia es una cuestión de énfasis y de marca de la institución, y forma parte de su cultura organizacional. No es un mito ni una moda: es una necesidad, una apuesta estratégica para afrontar con éxito el cambio, para organizar las bibliotecas de cara al futuro, que sean capaces de añadir valor a los proceso de enseñanza y aprendizaje, facilitando una sólida integración de la información digital en los entornos de trabajo de profesores, estudiantes e investigadores, así como en los procesos colaborativos de investigación. El cambio afecta también a los servicios de informática, que tendrán que adaptar sus 
aplicaciones a las nuevas necesidades y contextos. Ambos servicios se necesitan y deberían complementarse, pues la biblioteca precisa aplicaciones y herramientas suministradas por los centros de informática, y estos necesitan los contenidos que les suministran las bibliotecas.

La transformación al CRAI será más fácil en universidades jóvenes y de tamaño medio, donde es más sencillo hacer llegar los mensajes institucionales y donde el profesorado, en general es más joven y está dispuesto a los cambios. EI CRAI no es una imposición es una opción que cada universidad debe adaptar a su filosofía, a sus necesidades y a sus posibilidades. Es un proyecto de largo recorrido, que implica una actitud proactiva de los servicios convergidos y que irá madurando en su andadura con la implementación y evaluación de proyectos y servicios. (Pinto, 2008a, p.65)

La autora defiende que la transformación de biblioteca a CRAl es un trabajo bastante laborioso y que merita tiempo y dedicación. El Director del CRAl, debe ser una persona creativa, con mente abierta a los cambios, que sea líder dentro del colectivo, que logre la capacitación de todo su personal para poder llevar adelante este proceso. Porque los primeros que deben interiorizar la necesidad del cambio son los Especialistas en Información. Así como la integración de los diferentes perfiles profesionales. Para lograr la preparación de este proyecto, el director se debe dar a la tarea de realizar búsquedas sobre diferentes CRAI a nivel internacional; así como ejemplos de los mismos para resumir sobre los servicios, horarios de apertura y contenidos que ofrecen los mismos.

\section{Conclusiones}

Los Centros de Recursos Para el Aprendizaje y la Investigación (CRAI), permiten a los estudiantes participar en un proceso de enseñanza aprendizaje más dinámico, es decir la biblioteca se convierte en un centro dinamizador del aprendizaje, los apoya en la investigación y la docencia. Les propicia el trabajo con las diferentes fuentes de información y en diferentes formatos, al mismo tiempo les favorece el trabajo en grupo y los hace ser más autónomos, creativos e independientes.

Los CRAI, son una apuesta emergente y estratégica de las universidades para concentrar y rentabilizar sus servicios de apoyo a la comunidad universitaria. 


\section{Bibliografía}

Área Moreira, M.(2004) Informe Final de la Biblioteca Universitaria al Centro de Recursos Para el Aprendizaje e Investigación: Elaboración de una guía sobre la organización y gestión de un CRAl en el contexto de las Universidades Españolas. In. España: Ministerio de Educación y Ciencia. p. 608 Disponible en: http://es.scribd.com/doc/7177579/crai

Balagué Mola, N. (2003) La biblioteca universitaria, centro de recursos para el aprendizaje y la investigación: una aproximación al estado de la cuestión en España. In. Barcelona, España. p. 32 p. [Consultado 14 de abril de 2011]. Disponible en:

http://www.aab.es/pdfs/gtbu bu crai.pdf

Cabo Rigol, M. (2007) De la Biblioteca Universitaria Tradicional a los CRAI: Nuevos modelos organizativos. España: Universidad Pompeu Fabra; Disponible en: www.rebiun.org/export/docReb/w11.ppt

Domínguez Aroca, M. I.(2005) La biblioteca universitaria ante el nuevo modelo de aprendizaje: docentes y bibliotecarios juntos porque trabajamos juntos. RED. Revista de Educación a Distancia, IV(01V) Disponible en:

http://revistas.um.es/red/article/view/24481

Faros Para la Sociedad de La Información: (2005) Declaración de Alejandría sobre la Alfabetización Informacional y el aprendizaje a lo largo de la vida. In. Alejandría. p. 2 p. [Consultado 13 de abril de 2011]. Disponible en: www.coruna.es

Martín Gavilán, C. (2009) La edición-e y su impacto en las BU Acceso y gestión de los RE: los metadatos y los lenguajes de marcado. [Consultado 14 de junio 2011]. Disponible en: http://eprints. rclis.org/bitstream/10760/14565/1/edebu.pdf

Martínez, D. (2005) El Centro de Recursos Para el Aprendizaje CRAI. El nuevo modelo de biblioteca universitaria. In. Catalunya: Universidad Politécnica de Catalunya. p. 15 p. [Consultado 23 de noviembre de 2011]. Disponible en:

http://www.aab.es/pdfs/gtbu crai.pdf

McDonald, A. (2005) Creación de Centros de Recursos Para el Aprendizaje: Cualidades y recomendaciones Proyectos recientes en el Reino Unido. Bibliografía Selectiva. In. Madrid: REBIUN. p. 54. [Consultado 13 de Julio de 2011]. Disponible en:

http://www.rebiun.org/doc/z1.pdf

Maurial, N. M. K. D. (2005) Los Centros de Recursos para el Aprendizaje y la Investigación, CRAI. In. Perû. [Consultado 13 de abril de 2011]. Disponible en: http://eprints.rclis.org/bitstream/10760/9167/1/mackee crai.pdf

Nauto Díaz, S. (2006) Observando la Génesis del CRAI Un nuevo modelo de Biblioteca Universitaria. In. Barcelona, España: Valparaíso. [Consultado 13 de abril de 2011]. Disponible en: http://sibupla.upla.cl/sibupla1/archivos/UPF Barcelona.pdf

Pinto Molina, M., Sales, Dora., Osorio, Pilar. (2008) Biblioteca Universitaria, CRAl y Alfabetización informacional. TREA ed: Gijón, $245 \mathrm{p}$.

REBIUN. CRAI. (2012) Centro de Recursos Para el Aprendizaje y la Investigación. In: Docupedia: REBIUN; 2012. [Consultado 4 de enero 2012]. Disponible en: www.docupedia.es/articulo/crai-centro-de-recursos-para-el-aprendizaje-y-la-investigación

Ricci, M. A. (2009) Conferencia Mundial sobre Educación Superior 2009. "La nueva dinámica de la Educación Superior y la búsqueda del cambio social y el Desarrollo". In: Relexiones sobre Educación. Paris: UNESCO; 2009 Disponible en: http://miguelricci2008.blogspot.com/2010/01/conferencia-mundial-sobre-educacion.html

Serrat Brustenga, M. (2008) El Centro de Recursos para el aprendizaje y la investigación (CRAI) en permanente transformación: Servicios y Recursos para el nuevo usuario 2.0. In. Catalunya; 2008. [Consultado 14 de junio de 2011]. Disponible en: http://www.sedic.es/S3 C2 UPC.pdf

Taladriz Más, M. (2006) La biblioteca como centro de recursos: nuevos modelos para realidades cambiantes: Universidad Carlos III de Madrid Disponible en:

http://www.bne.es/es/Servicios/NormasEstandares/DocumentosProfesionales/BibliotecasyEducacion/Docs/060619 biblioteca com o centro de recursos.pdf

UNESCO. (1998) Declaración Mundial Sobre La Educación Superior en el Siglo XXI: Visión y Acción. In. París [Consultado 25 de junio de 2011]. Disponible en: http://www.unesco.org/education/educprog/wche/declaration spa.htm 


\section{Datos del autor}

Raquel Zamora Fonseca

Máster en Educación. Máster en Bibliotecología y Ciencias de la Información. Directora del Centro de Recursos para el Aprendizaje de la Universidad de Cienfuegos.

araimis@correo.ugr.es

Recibido-Received : 2012-10-22

Aceptado-Accepted : :2012-12-30

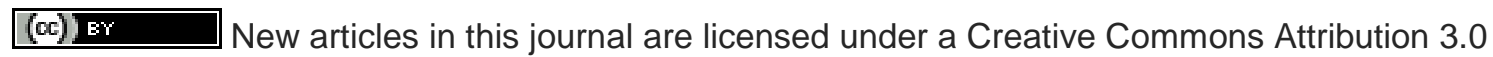
United States License.

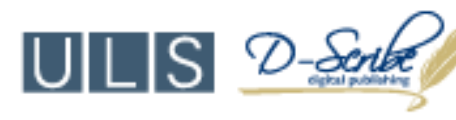

This journal is published by the University Library System of the University of Pittsburgh as part of its D-Scribe Digital Publishing Program and is cosponsored by the University of Pittsburgh Press. 